\title{
A theoretical and experimental study of the time-dependent radiative properties of a solar bubbling fluidized bed receiver
}

\author{
G. Baud ${ }^{\mathrm{a}, *}$, J.J. Bezian ${ }^{\mathrm{a}}$, M. El Hafi ${ }^{\mathrm{a}}$, G. Olalde ${ }^{\mathrm{b}}$ \\ a Centre RAPSODEE, ENSTIMAC, Campus Jarlard, 81013 Albi CT Cédex 09, France \\ ${ }^{\mathrm{b}}$ PROMES-CNRS, 7 rue du Four Solaire, 66120 Font Romeu Odeillo, France
}

\begin{abstract}
In order to evaluate the potential of solar bubbling fluidized bed receivers compared to other methods for the solar heating of gases at high temperature, a thorough knowledge of the heat transfer of the receiver is necessary. Since the external energy source of the system is radiative and because of high working temperatures, it is particularly important to model the radiative heat transfer to later predict the temperature field in the solar receiver. The aim of this study is to model the radiative flux distribution in a fluidized bed by taking into account the time-dependent absorption and scattering of light in the particulate medium. For this purpose, we propose a model using the Monte Carlo Method as well as a time-dependent field of optical properties that was predicted using a Computational Fluid Dynamics tool implemented with an Eulerian model. A statistical treatment using the $k$-distribution method was later applied to the time-dependency of the radiative properties of the solar fluidized bed receiver. This method has proven to be useful to reduce computational time while keeping a good accuracy. An experimental set-up was designed to validate the numerical predictions of the particle volume fraction and the penetration of radiation into the fluidized bed. The good agreement of the current model with the experimental data confirms its suitability.
\end{abstract}

Keywords: Bubbling fluidized bed; Radiative properties; Solar receiver; Monte Carlo Method; $k$-Distribution method; Time-dependent calculation

\section{Introduction}

Fluidized beds are conventionally used as media of thermal exchanges for high temperature processes because of their excellent performance in terms of heat transfer. To improve the performance of thermodynamic cycles for solar electricity production and to obtain temperatures above $1000 \mathrm{~K}$ at the entrance of a turbine, technological gaps involving direct gas heating in solar receivers would need to be bridged. One of the best solutions would be to

\footnotetext{
* Corresponding author. Tel.: +33563767141.

E-mail address: germain.baud@mines-albi.fr (G. Baud).
}

use solid particles in a fluidized bed that are directly exposed to the concentrated solar flux. The main advantages of the fluidized bed receiver are its thermal efficiency, an improved resistance to thermal stress compared to metallic and ceramic receivers and its adaptability to beam down concentrators that allow producing electricity directly on the ground. For these reasons, fluidized bed solar receivers and reactors were designed and studied by numerous scientists: Flamant and Olalde (1983), Haddad and Elsayed (1988), Muller et al. (2003), Trommer et al. (2005) and Chen et al. (2004). However, even nowadays, some technical issues are still not well understood such as the high radiative losses of the system and the appearance 


\begin{tabular}{|c|c|c|c|}
\hline \multicolumn{4}{|c|}{ Nomenclature } \\
\hline$\rho$ & density $\left(\mathrm{kg} \mathrm{m}^{-3}\right)$ & $E$ & absorbed fraction of incoming light \\
\hline$\alpha$ & volume fraction $(-)$ & $S_{0}$ & surface of the radiation source $\left(\mathrm{m}^{2}\right)$ \\
\hline$\Delta P$ & pressure drop $(\mathrm{Pa})$ & $u_{0}$ & radiative emission direction \\
\hline$\Delta H$ & height (m) & $P$ & random point on $S_{0}$ \\
\hline$\vec{g}$ & gravity $\left(\mathrm{m} \mathrm{s}^{-2}\right)$ & $\gamma$ & optical path originated from $P$ in the direction \\
\hline$\vec{v}$ & instantaneous velocity vector $\left(\mathrm{m} \mathrm{s}^{-1}\right)$ & & $u_{0}$ \\
\hline$\overline{\bar{\tau}}$ & phase stress-strain tensor $(\mathrm{Pa})$ & $l_{\gamma, n}^{-}$ & $n$th entry point coordinates of optical path $\gamma$ in \\
\hline$K_{g s}$ & gas/solid momentum exchange $\left(\mathrm{kg} \mathrm{m}^{-3} \mathrm{~s}\right)$ & & gas volume elements \\
\hline$\mu$ & viscosity (Pa s) & $l_{\gamma, n}^{+}$ & $n$th exit point coordinates of optical path $\gamma$ in \\
\hline$\stackrel{\lambda}{=}$ & bulk viscosity $\left(\mathrm{kg} \mathrm{s}^{-1} \mathrm{~m}\right)$ & & gas volume elements \\
\hline$\overline{\bar{I}}$ & unity matrix & $\sigma$ & curvilinear abscissa in the $n$th intersection inter- \\
\hline$C_{D}$ & drag coefficient $(-)$ & & val between $\gamma$ and gas volume elements \\
\hline $\mathrm{Re}$ & Reynolds number (-) & \multirow{2}{*}{\multicolumn{2}{|c|}{$\begin{array}{c}p\left(\gamma ; P, u_{0}\right) \text {, distribution function representing the exis- } \\
\text { tence probability of a given optical path } \gamma\end{array}$}} \\
\hline$p_{s}$ & solid pressure $(\mathrm{Pa})$ & & \\
\hline$\theta_{s}$ & granular temperature & $\Gamma_{\left(P, u_{0}\right)}$ & space of optical paths $\gamma$ originated from $P$ in the \\
\hline$e_{s s}$ & restitution coefficient $(-)$ & & direction $u_{0}$ \\
\hline$g_{0, s s}$ & radial distribution coefficient $(-)$ & $T_{\gamma, n}$ & transmissivity of the optical path $\gamma$ from $P$ to $l_{\gamma, n}^{-}$ \\
\hline$\mu_{s, \mathrm{col}}$ & collisional viscosity (Pa s) & $\Delta t$ & time bandwidth $(\mathrm{s})$ \\
\hline$\mu_{s, \text { kin }}$ & kinetic viscosity $(\mathrm{Pa} \mathrm{s})$ & $\Phi$ & a given function \\
\hline $\begin{array}{l}\mu_{s, \mathrm{fri}} \\
d\end{array}$ & $\begin{array}{l}\text { frictional viscosity (Pa s) } \\
\text { diameter }(\mathrm{m})\end{array}$ & $f$ & distribution function \\
\hline$\gamma_{\theta s}$ & collisional dissipation energy $\left(\mathrm{kg} \mathrm{s}^{-3} \mathrm{~m}\right)$ & \multicolumn{2}{|c|}{ Subscripts } \\
\hline$\phi_{g s}$ & transfer rate of kinetic energy $\left(\mathrm{kg} \mathrm{s}^{-3} \mathrm{~m}\right)$ & $s$ & solid or scattering \\
\hline$g$ & gravity $\left(\mathrm{m} \mathrm{s}^{-2}\right)$, HG phase function coefficient & $g$ & \\
\hline$O$ & $\begin{array}{l}\text { or cumulative distribution function } \\
\text { ontical efficiency }\end{array}$ & $a$ & absorption \\
\hline
\end{tabular}

of hot spots on the walls of the receiver under high solar flux.

To evaluate the potential of the fluidized bed solar receiver and address its weaknesses, it is necessary to model heat transfer and calculate the temperature field in the solar receiver. Since the external energy source is radiative and because of high working temperatures, it is particularly important to model radiative heat transfer to later predict the temperature field in the solar receiver and the thermal fluxes on the walls of the receiver.

Several models have been used to predict radiative properties of fluidized particles and estimate radiative heat transfer in fluidized beds. Two major types of methods to determine radiative properties were used. The first one is based on mathematical or empirical steady-state radiative properties of the fluidized bed. In this category, we can name Gordillo and Belghit (2010) or Mendes et al. (2008) using the Rosseland approximation, Von Zedtwitza et al. (2006) or Hua et al. (2004) using the Monte Carlo Method (MCM), Selçuk et al. (2002) using the Discrete Ordinates Method (DOM) and Arancibia-Bulnes and Cuevas (2004) using the P1 method. The second one is based on timedependent radiative properties that are calculated with the help of Computational Fluid Dynamics (CFD) tools. In this category, we refer to the work of Reuge et al.
(2009) using the Rosseland approximation, Lathouwers and Bellan (2001) using the six flux method or Klein et al. (2007) using the MCM method.

However, only few studies paid attention to both the time-dependence of the radiative properties of the fluidized bed and the multiple scattering of light in the particulate medium. For this reason, this paper focuses on determining the time-dependent radiative properties of bubbling fluidized beds to allow the calculation of the radiative source term. The Radiative Transfer Equation (RTE) is solved through its integral formulation using the MCM, which takes into account the absorption and the multiple scattering of light in the particulate medium. An experimental setup was designed to validate the numerical predictions of the particle volume fraction and the penetration of radiation into the fluidized bed at room temperature.

\section{Modeling methodology}

\subsection{Modeling of particle volume fraction}

\subsubsection{Modeling strategy}

To our knowledge, no commercial CFD tool is capable of predicting flow fields, temperature distributions as well as convective and radiative fluxes simultaneously and 
accurately in bubbling fluidized beds. As an instance, the DOM used in ANSYS Fluent is based on an angular discretization of the space. As a consequence, the space need to be discretized with a very high number of directions, which increase calculation time without being even sure to capture the fine radiative effects leading to the appearance of hot spots on the walls of the receiver.

In order to address these problems, the CFD tool is only used for predicting the time dependent volume fraction without solving the energy conservation. The volume fraction is later used to compute the radiative properties of the fluidized bed and the radiative fluxes. Not solving the energy equations is viable considering that the fluidized bed in this study is operated at room temperature and that the radiative source is a low power laser diode that has a negligible thermal impact on the fluidized bed.

However, when operating a real solar fluidized receiver, the point was made that temperature can have an important effect on the fluidized bed hydrodynamics as shown in the work of Chaouki et al. (2003), Kunii et al. (1973), Furosaki and Kai (1985) or Mancuso et al. (1997). As a consequence, it would be necessary to take the effect of temperature into account for solving the energy conservation equations. As a simplified approach, we can also assume that both particle and fluid temperatures in the fluidized bed are fixed and homogeneous in order to solve the hydrodynamics modeling equations. The hypothesis that no thermal gradient occurs in the fluidized bed is reasonable in most of the bed because of the high value of the heat transfer coefficient and the vigorous mixing of particles (Donnadieu, 1992) (Cf. Fig. 1). However, this hypothesis is false in the first few centimeters at the bottom because of the cold gas inlet and at the top of the fluidized bed because of the high solar flux. The temperature of the bed is defined by the overall energy balance of the receiver. This simplified approach is valuable in this particular case, where only the distribution of concentrated light into the

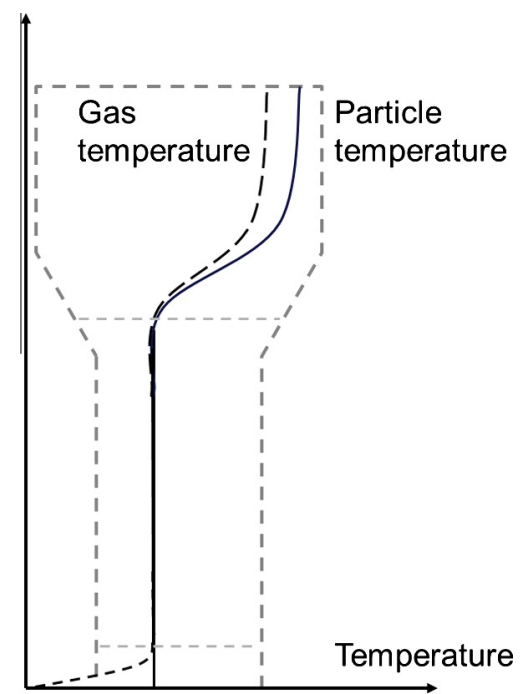

Fig. 1. Simplified temperature distribution in a solar fluidized bed receiver. receiver is computed in order to predict the appearance of hot spots on the walls.

\subsubsection{Multiphase flow modeling}

The particle volume fraction is calculated using an Eulerian multiphase model implemented in Fluent 12 (Ansys, 2009). A previous study comparing numerical results with our observations from experimental studies detailed in Baud, 2011 allowed us to make the following assumptions and set the calculation parameters so as to guarantee a good accuracy of the results within a reasonable calculation time. The governing equations are as follows:

- Conservation of mass:

$\frac{\partial}{\partial t}\left(\alpha_{g} \rho_{g}\right)+\nabla \cdot\left(\alpha_{g} \rho_{g} \overrightarrow{v_{g}}\right)=0$

$\frac{\partial}{\partial t}\left(\alpha_{s} \rho_{s}\right)+\nabla \cdot\left(\alpha_{s} \rho_{s} \overrightarrow{v_{s}}\right)=0$

- Conservation of momentum:

$$
\begin{aligned}
& \frac{\partial}{\partial t}\left(\alpha_{g} \rho_{g} \overrightarrow{v_{g}}\right)+\nabla \cdot\left(\alpha_{g} \rho_{g} \overrightarrow{v_{g}} \overrightarrow{v_{g}}\right) \\
& \quad=-\alpha_{g} \nabla p+\nabla \cdot\left(\overline{\overline{\tau_{g}}}\right)+\alpha_{g} \rho_{g} \vec{g}-K_{g s}\left(\overrightarrow{v_{g}}-\overrightarrow{v_{s}}\right) \\
& \frac{\partial}{\partial t}\left(\alpha_{s} \rho_{s} \overrightarrow{v_{s}}\right)+\nabla \cdot\left(\alpha_{s} \rho_{s} \overrightarrow{v_{s}} \overrightarrow{v_{s}}\right) \\
& \quad=-\alpha_{s} \nabla p-\nabla p_{s}+\nabla \cdot\left(\overline{\overline{\tau_{s}}}\right)+\alpha_{s} \rho_{s} \vec{g}+K_{g s}\left(\overrightarrow{v_{g}}-\overrightarrow{v_{s}}\right)
\end{aligned}
$$

with

$\overline{\overline{\tau_{g}}}=\mu_{g}\left(\nabla \overrightarrow{v_{g}}+\nabla \overrightarrow{v_{g}} T\right)+\left(\lambda_{g}-\frac{2}{3} \mu_{g}\right) \nabla \overrightarrow{v_{g}} \overline{\bar{I}}$

$\alpha_{s} \overline{\overline{\tau_{s}}}=-p_{s} \overline{\bar{I}}+\alpha_{s} \mu_{s}\left(\nabla \overrightarrow{v_{s}}+\nabla{\overrightarrow{v_{s}}}^{T}\right)+\left(\lambda_{s}-\frac{2}{3} \mu_{s}\right) \nabla \overrightarrow{v_{s}} \overline{\bar{I}}$

- Inter-phase momentum transfer by Di Felice (1994):

$K_{g s}=\frac{3 C_{D} \alpha_{s} \rho_{g}}{4 d_{s}} f\left(\alpha_{s}\right)$

with

$f\left(\alpha_{s}\right)=\left(1-\alpha_{s}\right)^{-x}$

$\left.x=P-Q \exp -\frac{(1.5-\beta)^{2}}{2}\right)$

$\beta=\log _{10}\left(\operatorname{Re}_{s}\right)$

$P=3.7$ and $Q=0.65$ being experimentally adjusted

- Constitutive equations:

- Solid pressure by Lun et al. (1984):

$p_{s}=\alpha_{s} \rho_{s} \theta_{s}+2 \rho_{s}\left(1+e_{s s}\right) \alpha_{s}^{2} g_{0, s s} \theta_{s}$

- Radial distribution function by Sinclair and Jackson (1989):

$g_{0, s s}=\left[1-\left(\frac{\alpha_{s}}{\alpha_{s, \max }}\right)^{1 / 3}\right]^{-1}$ 
- Bulk viscosity by Lun et al. (1984):

$\lambda_{s}=\frac{4}{3} \alpha_{s} \rho_{s} d_{s} g_{0, s s}\left(1+e_{s s}\right)\left(\frac{\theta_{s}}{\pi}\right)^{1 / 2}$

- Solid shear stress:

$\mu_{s}=\mu_{s, \mathrm{col}}+\mu_{s, \mathrm{kin}}+\mu_{s, \mathrm{fri}}$

- Collisional viscosity:

$\mu_{s, \mathrm{col}}=\frac{4}{5} \alpha_{s} \rho_{s} d_{s} g_{0, s s}\left(1+e_{s s}\right)\left(\frac{\theta_{s}}{\pi}\right)^{1 / 2}$

- Kinetic viscosity by Syamlal et al. (1993):

$\mu_{s, \mathrm{kin}}=\frac{\alpha_{s} \rho_{s} d_{s} \sqrt{\theta_{s} \pi}}{6\left(3-e_{s s}\right)}\left[1+\frac{2}{5}\left(1+e_{s s}\right)\left(3 e_{s s}-1\right) \alpha_{s} g_{0, s s}\right]$

- Frictional viscosity by Schaeffer (1987):

$\mu_{s, \mathrm{fri}}=\frac{P_{s} \sin \phi}{2 \sqrt{I_{2 D}}}$

- Granular temperature:

$\frac{3}{2}\left[\frac{\partial}{\partial t}\left(\alpha_{s} \rho_{s} \theta_{s}\right)+\nabla \cdot\left(\alpha_{s} \rho_{s} \overrightarrow{v_{s}} \theta_{s}\right)\right]=\left(-p_{s} \overline{\bar{I}}+\overline{\overline{\tau_{s}}}\right)$

$: \nabla \overrightarrow{v_{s}}+\nabla\left(k_{\theta s} \nabla \theta_{s}\right)-\gamma_{\theta s}+\phi_{g s}$

with

$k_{\theta s}=\frac{15 d_{s} \alpha_{s} \rho_{s} \sqrt{\theta_{s} \pi}}{4(41-33 \eta)}\left[1+\frac{12}{5} \eta^{2}(4 \eta-3) \alpha_{s} g_{0, s s}+\frac{16}{15 \pi}(41-33 \eta) \eta \alpha_{s} g_{0, s s}\right]$

$\eta=\frac{1}{2}\left(1+e_{s s}\right)$

$\gamma_{\theta s}=\frac{12\left(1-e_{s s}^{2}\right) g_{0, s s}}{d_{s} \sqrt{\pi}} \rho_{s} \alpha_{s}^{2} \theta_{s}^{3 / 2}$

$\phi_{g s}=-3 K_{g s} \theta_{s}$

Calculations are 3D and time-dependent. The time step size used in the numerical simulations is of the order of magnitude of $1 \mathrm{~ms}$. Particles are considered spherical and monodisperse. The monodisperse assumption has been verified viable in order to represent the sifted particles used in the experimental setup. To prove that the particle size distribution has only a weak influence on both flow characteristics and radiative calculations, we first proceeded to the CFD calculation of a fluidized bed using either a monodisperse particle size distribution or a 3 phase diameter distribution modeling the experimental diameter distribution. Neither diameter segregation nor flow differences were noted. Later, we evaluated the radiative properties on both a monodisperse distribution and a log-normal distribution fitting the experimental data. It appears that the little difference in radiative properties did not influence the radiative calculations. Attrition is neglected as the time of experiment is kept short. A standard extended $k-\varepsilon$ model extended to multiphase flows is used. At the bottom of the column, the gas input is considered at a homogeneous velocity. The walls of the receiver have a no-slip condition. The mesh size is fixed at around 10 times the particle size.

When the steady state is reached after $20 \mathrm{~s}$ of calculation, the particle volume fraction is read at a frequency of $1 \mathrm{~Hz}$ over $10 \mathrm{~s}$ in different points aligned on the central axis and positioned every half-centimeter from the bottom to the top of the receiver.

\subsection{Radiative heat transfer modeling}

\subsubsection{Radiative transfer in bubbling fluidized beds}

When a particle bed is exposed to radiation, mechanisms such as absorption and scattering contribute to the extinction of radiative transfer along the fluidized bed. The spreading of radiation occurs through the interstices of the particle fluidized bed by transmission and scattering due to the particles. In this work, independent scattering is considered as the particles, fluidization and light parameters fulfill the Tien and Drolen criterium for independent and dependent scattering (Tien and Drolen, 1987). The air is transparent to radiation. The penetration depth depends largely on the geometry of the receiver, the volume fraction of the fluidized bed and the optical properties of the particles.

\subsubsection{Radiative properties of the particles in the receiver}

The absorption and scattering coefficients depend on the volume fraction and the optical properties of the particles. The description of the scattering in the particle cloud is modeled using the Henyey-Greenstein phase function. To determine the optical properties of the particles, a calculation over the whole solar spectrum (between $0.24 \mu \mathrm{m}$ and $4 \mu \mathrm{m})$ is carried out using a code which is based on Mie theory (Mie, 1908) developed by Mishchenko.

Even if the size parameter calculated for this particular study is greater than 1 and thus suggests the use of optical geometry, the Mie theory was chosen in order to later extend the methodology subsequently to smaller particle diameters and shorter wavelengths of light.

In the case of $\mathrm{SiC}$ particles, previous results have shown that the dependency of the radiative properties on the wavelength is not significant in the solar spectrum (Baud et al., 2012). From the complex index of refraction of $\mathrm{SiC}$ particles found in the Handbook of optical constants of solids (1985), we can thus calculate averaged values of optical properties $Q_{s}, Q_{a}$ and $g$. Subsequently, we performed grey calculations where the optical properties are described by the following values of the absorption and scattering cross sections:

$k_{a}=\frac{3}{2} \frac{\alpha_{s} Q_{a}}{d_{s}}$

$d_{s} k_{s}=\frac{3}{2} \frac{\alpha_{s} Q_{s}}{d_{s}}$

With $Q_{a}=0.5, Q_{s}=1.51$ and $g=0.7$ for $280 \mu \mathrm{m} \mathrm{SiC}$ particles. 
The stainless steel surface of the receiver is represented by a diffuse-reflective surface with a grey reflectivity of 0.6. The quartz window is considered to be a specularly diffuse and transmissive surface with a reflectivity that depends on the incident angle. The air within the receiver is transparent.

\subsubsection{A 3D radiative transfer model using the Monte Carlo Method}

The RTE (Radiative Transfer Equation) is solved by its integral formulation. The MCM (Monte Carlo Method) (Metropolis and Ulam, 1949) is used to explicitly calculate these integrals (Coelho et al., 2003; Joseph et al., 2009; De Lataillade et al., 2002). As a statistical method, the MCM produces reference solutions in the sense that solutions are provided with their corresponding standard deviation. The resolution of the RTE using the MCM allows us to study, without approximation both optically thin (bubbles) and optically thick (emulsion phase) media found in a bubbling fluidized bed. To calculate the penetration depth in the fluidized bed, absorption and scattering are taken into account.

The fluidized bed is discretized into surface and volume elements of homogeneous radiative properties. The fraction of incoming solar energy absorbed in each volume element is calculated by:

$\left.E_{j}=\int_{S_{0}} d S_{0}(P) \int_{\left.\Gamma_{(} P, u_{0}\right)} p\left(\gamma ; P, u_{0}\right) d \gamma \sum_{n=1}^{\infty} T_{\gamma, n} \quad 1-\exp \left(-\int_{l_{\gamma, n}^{-}}^{l_{\gamma, n}^{+}} k_{a}(\sigma) d \sigma\right)\right)$

The associated algorithm derived from this formulation may be described as:

- A point $P$ is randomly chosen on the surface $S_{0}$ of the radiation source and a ray is emitted in the direction $u_{0}$.

- Starting from the point $P$, an optical path $\gamma$ is generated according to a standard random walk technique in a diffusive medium.

- Each time that $\gamma$ reaches a volume or a boundary, its extinction and its thermal contribution are calculated.

- Finally, the optical path $\gamma$ ends when it exits the receiver or when it is long enough for the energy bundle to be considered totally attenuated.

The fraction of incoming solar energy absorbed by each surface element on the walls of the receiver is calculated in a similar manner not described in the present paper.

The radiative model is implemented in the EDStaR environment (Delatorre et al., 2013). This environment allows for the use of computer graphics libraries to efficiently calculate intersections between beams and any wall surfaces. It is also implemented with parallel computing tools and specific functionalities to produce statistical quantities and their derivatives.

\subsubsection{Calculation of the radiative transfer from numerical particle volume fraction}

As pointed out earlier, radiative properties within the receiver depend on the particle volume fraction in the fluidized bed. The receiver is discretized into $1 \mathrm{~cm}$ wide layers in which the radiative properties have been spatially averaged. Mesh independence has been checked. As there are different methods to determine the particle volume fraction from the CFD calculations, there is also more than one way to calculate the time-averaged repartition of radiation inside the fluidized bed receiver. For the purpose of our study, three alternatives were selected and compared:

- The first alternative is to compute the radiative transfer in the fluidized bed from the time-averaged optical properties (i.e. time-averaged particle volume fraction). The particle volume fraction is averaged over $10 \mathrm{~s}$ of CFD simulation when the steady state is reached.

- The second alternative is to compute the radiative transfer in the fluidized bed at different time steps to finally average these calculations over the specified time. In this paper, the radiative calculation is carried out at a frequency of $10 \mathrm{~Hz}$ over $10 \mathrm{~s}$. As a result, 100 calculations are performed and averaged to produce the final result.

- As a third alternative, we propose to calculate the radiative transfer based on a statistical treatment of the time-dependent radiative properties. We decided to apply the $k$-distribution approach to reorder the absorption coefficient depending on time. This idea is based on the signal shape similarity between the wavelengthdependent distribution of the radiative properties of a gas and the time-dependent distribution of the radiative properties of a bubbling fluidized bed.

\subsubsection{The statistical treatment: The k-distribution method}

Looking at Fig. 2, the shape of the particle volume fraction versus time (proportional to the absorption coefficient of the medium) has strong similarities with the absorption coefficient versus wavelength in gas radiative species. Even if this analogy cannot be physically based, we apply the same procedure relying on statistical treatment to these similar signals: reordering $k$ using the distribution of $k$ and its cumulative.

The $k$-distribution method (first introduced in Ambartzumian (1936)) consists of subdividing the entire spectrum into narrow-bands of width $\Delta t$ and then solving the radiative transfer over each narrow-band. To integrate the radiative quantities inside a narrow band, two variables are defined. The first one is the distribution function $f$ which is defined in such a way that the source represents the fraction of $\Delta t$ where the absorption (or scattering) coefficient lies between $k$ and $k+d k$. The other one is the cumulative distribution function $g$ of the absorption coefficient, which represents the probability of the absorption 

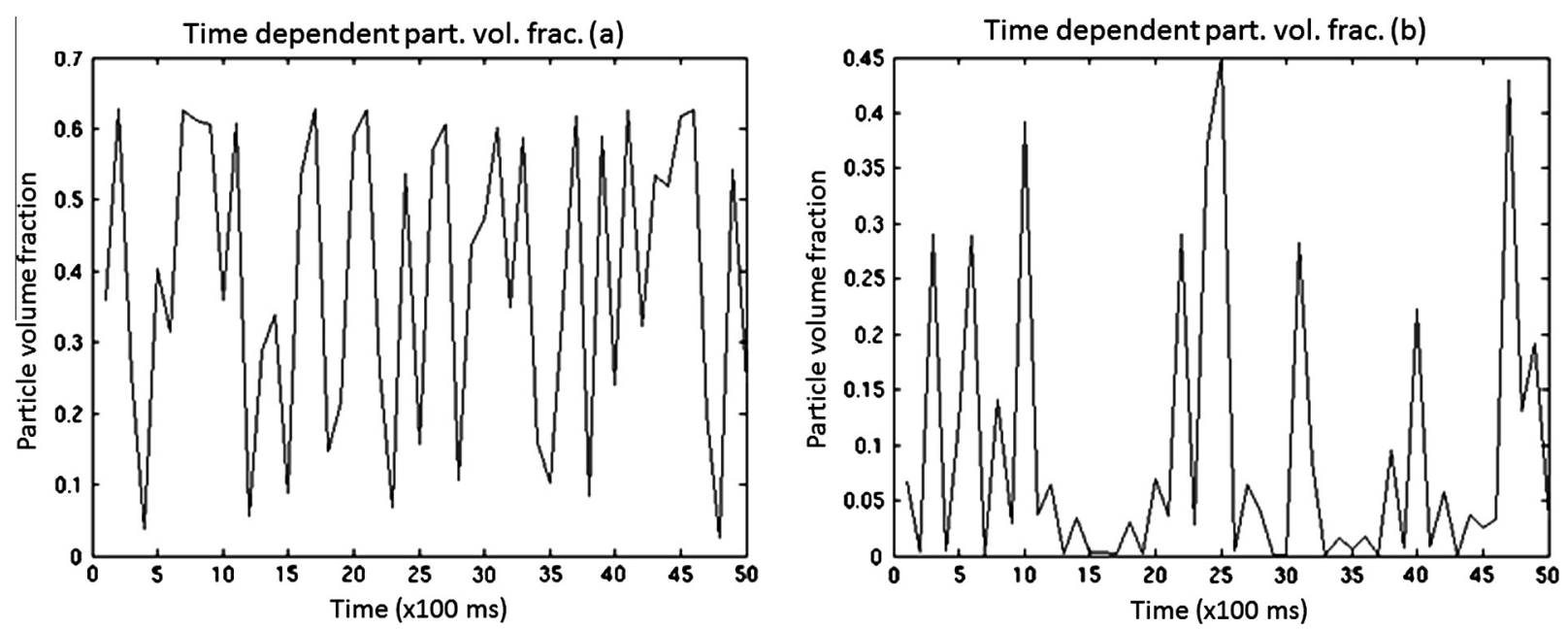

Fig. 2. Time-dependent evolution of the particle volume fraction for the case H19N3.4 as described in Section 4.1: measured (a) near the bottom and (b) near the top of the fluidized bed.

(or scattering) coefficient to be less than $k$ of the bandwidth $\Delta t$ and which can be interpreted as pseudo-time varying between 0 and 1 . Thus, the average distribution of radiative energy in the fluidized bed can be defined by analogy of the wavelength $\lambda$ to the time variable $t$ :

$$
\begin{aligned}
\frac{1}{\Delta t} \int_{\Delta t} \Phi(t) & =\int_{0}^{\infty} \Phi(k) f(k) d k \\
& =\int_{0}^{1} \Phi(k(g)) d g \approx \sum_{j=0}^{N_{q}} \omega_{j} \Phi\left(k\left(g_{i}\right)\right)
\end{aligned}
$$

The main advantage expected when using this model is minimizing the number of computations required for the transient radiative calculations and thus reducing computational time. From the large number of time-step calculations necessary to calculate the averaged radiation distribution, only a limited number of calculations according to quadrature points are required. For this study, we applied the Legendre quadrature (of the 16th order).

\section{Description of the experimental set-up}

The solar fluidized bed receiver (cf. Fig. 3) consists of a gas diffuser, a lower cylindrical column containing particles of silicon carbide and a higher cylinder-conical column surmounted by a quartz window. The receiver is $45 \mathrm{~cm}$ high with a lower part measuring $25 \times 7 \mathrm{~cm}$, a middle conical part of $7.5 \mathrm{~cm}$ and an upper part of $12.5 \times 12 \mathrm{~cm}$. The gas that is sent through a metallic diffuser at the bottom of the column supports the bubbling fluidization of silicon carbide $(\mathrm{SiC})$ particles. The rise of diameter at the top of the receiver prevents the particles from leaving the fluidization column.

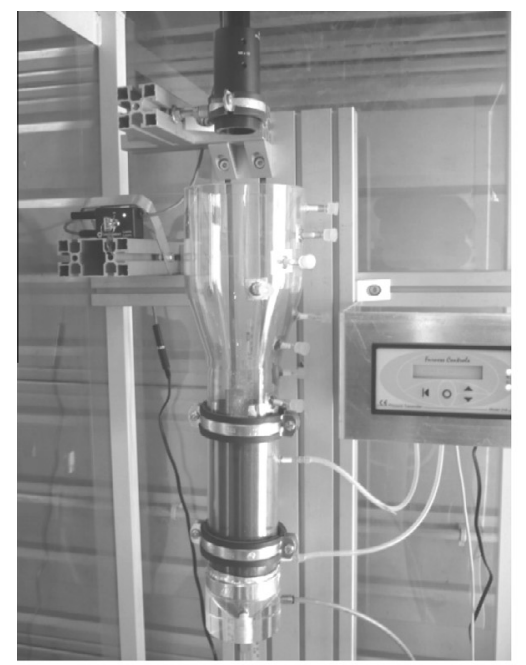

(a)

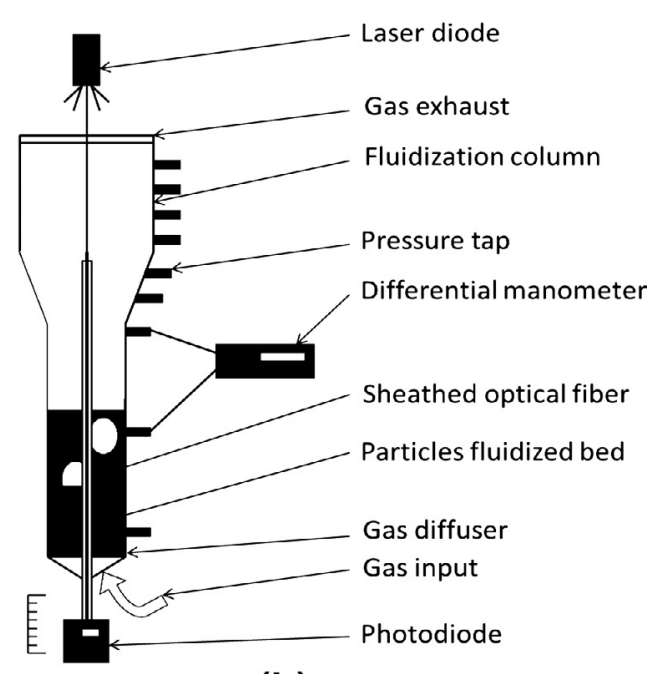

(b)

Fig. 3. Picture (a) and scheme (b) of a $45 \mathrm{~cm}$ high fluidized bed receiver with a lower part measuring $25 \times 7 \mathrm{~cm}$, a middle conical part of $7.5 \mathrm{~cm}$ and an upper part of $12.5 \times 12 \mathrm{~cm}$. 
The measuring instruments implemented in the experimental set-up can be divided into two categories that are designed to validate the numerical calculations:

- The optical assembly that allows for the measurement of the transmitted radiative flux at the bottom of the fluidized bed.

- The pressure assembly that allows for the calculation of the mean particle volume fraction at different sections of the bed.

\subsection{Optical assembly}

At the top of the receiver, there is a collimated laser diode with a beam expander that is directed toward the bed. The red beam emitted by the laser diode is $4 \mathrm{~cm}$ wide. Inside the receiver there is a $1 \mathrm{~mm}$ multimode optical fiber sheathed and rigidified by a $5 \mathrm{~mm}$ glass tube. The optical fiber can be moved along the central axis of the receiver at different heights and propagates the radiation that is collected by a photodiode (cf. Fig. 2).

Moving the sensor along the central axis of the receiver allows us to measure the transmitted radiative flux in the fluidized bed. The data sampling is carried out with up to $1 \mathrm{MHz}$ and a precision of $10 \mathrm{pW}$. The maximum power received by the diode is $281 \mu \mathrm{W}$ corresponding to a $100 \%$ transmission of the radiation. The radiative flux on the central axis of the receiver can be measured every millimeter from the top until the complete extinction of the laser beam.

The data measurements are obtained when the fluidized bed is in steady state. The mass and diameter distribution of particles is checked before and after each measurement campaign. For each position, the radiative flux is measured over a minute to compute an averaged value. The percentage of transmitted radiative flux inside the particle cloud is calculated from the measurement of the radiative flux at different depths and is corrected by a calibration which is performed on an empty fluidization column.

A statistical study which was carried out over the course of a few days consisting of about 20 repetitions of each measurement has demonstrated a relative error of around $2.5 \%$ with respect to the transmitted flux.

\subsection{Pressure assembly}

Ten pressure taps are placed along the fluidization column. These pressure taps are made as small and sharp as possible in order to achieve the best accuracy possible. The distance between each pressure tap varies from 12 to $3 \mathrm{~cm}$ to insure a better resolution where a variation of the particle concentration is important (cf. Fig. 2).

The pressure drop between two pressure taps is measured using a differential manometer. The accuracy of the manometer is $5.6 \mathrm{~Pa}$ in the $250-2500 \mathrm{~Pa}$ range for a sampling frequency of $10 \mathrm{~Hz}$. The data measurements are obtained in the steady-state condition measured during one minute at each point.

The mean particle volume fraction between two pressure taps is calculated from the mean pressure drop using the relationship described in Rensner et al. (1986):

$f_{v}=\frac{\Delta P}{\Delta H\left(\rho_{P}-\rho_{g}\right) g}$

where $\Delta P$ stands for the pressure drop and $\Delta H$ is the height between two pressure taps.

A statistical study which was carried out over the course of a few days consisting of about 20 repetitions of each measurement demonstrated a maximum relative error of around $3 \%$ in the particle volume fraction.

\section{Results}

\subsection{Calculation parameters and description of the test cases}

Three test cases were studied using the $45 \mathrm{~cm}$ high receiver. These cases, described in Table 1, are all featuring sifted $280 \mu \mathrm{m} \mathrm{SiC} \mathrm{Geldart} \mathrm{B} \mathrm{particles} \mathrm{under} \mathrm{different} \mathrm{fluid-}$ ization parameters: the height of particles of the initial fixed bed and the number of fluidization. The fluidization number represents the ratio of the gas velocity in the column to the initial fluidization rate of particles (i.e. minimum velocity to reach fluidization). The $\mathrm{SiC}$ particle density is $3200 \mathrm{~kg} \mathrm{~m}^{-3}$.

Fluidization parameters are chosen to maintain the splashing zone of the bubbling fluidized bed in the conical section of the receiver where the concentrated sun radiation is expected to hit the particles. These fluidization parameters also provide an efficient turbulent mixing in the fluidized bed while preventing particles from exiting the fluidization column.

\subsection{Comparison of numerical and experimental particle volume fraction}

Since radiative properties within the receiver derive from the particle volume fraction in the fluidized bed, it is important to validate the capability of the CFD model to describe the hydrodynamics of the fluidized bed.

The particle volume fractions, that are either calculated using CFD tools or processed from the measurement of the pressure drop on the experimental set-up, are compared. The volume fractions are averaged over $10 \mathrm{~s}$. The different slices of the fluidized bed studied are defined to match the experimental pressure taps (Ex: Slice $X-Y \mathrm{~cm}$ stands for the

Table 1

Presentation of the different test cases.

\begin{tabular}{lll}
\hline Name of the case & Particle height: $H(\mathrm{~cm})$ & Number of fluidization: $N$ \\
\hline Case H19N3.4 & 19 & 3.4 \\
Case H16N2 & 16 & 2 \\
Case H12N3 & 12 & 3 \\
\hline
\end{tabular}


Table 2

Comparison of experimental and calculated averaged particle volume fractions for the case H19N3.4.

\begin{tabular}{lllll}
\hline & Slice & Slice & Slice & Slice \\
& $3-15 \mathrm{~cm}$ & $15-22 \mathrm{~cm}$ & $22-25 \mathrm{~cm}$ & $25-28 \mathrm{~cm}$ \\
\hline Experimental & 0.41 & 0.32 & 0.21 & 0.1 \\
CFD & 0.42 & 0.42 & 0.28 & 0.11 \\
\hline
\end{tabular}

Table 3

Comparison of experimental and calculated relative errors in percent of the averaged particle volume fraction.

\begin{tabular}{lllll}
\hline & Slice & Slice & Slice & Slice \\
& $3-15 \mathrm{~cm}$ & $15-22 \mathrm{~cm}$ & $22-25 \mathrm{~cm}$ & $25-28 \mathrm{~cm}$ \\
\hline Case H19N3.4 & 2.4 & 31.3 & 33.3 & 10 \\
Case H16N2 & 4.2 & 14.8 & 20 & NA \\
Case H12N3 & 1.7 & 6.3 & 10 & NA \\
\hline
\end{tabular}

height between $X$ and $Y$ ). Table 2 introduces the experimental and calculated particle volume fraction for the case H19N3.4.

Table 3 introduces the relative errors between the experimental and numerical particle volume fractions for all three cases. NA (Not Applicable) corresponds to data where the particle volume fraction is too low to be determined because of the imprecision of the measurements.

Comparing experimental and CFD results, we noticed that the relative error in the particle volume fraction can be as low as $1.7 \%$ and does not exceed $34 \%$. As the error in the particle volume fraction is proportionally reflected in the calculated radiative properties, a similar error can be expected in the calculation of the absorption and scattering coefficients in the fluidized bed.

Even if the effect of this error is important for the calculation of the radiative transfer, its impact on the calculation of the temperature field in the fluidized bed is reduced due to high thermal mixing in the fluidized bed. Thus, the CFD tool is considered suitable for predicting the particle volume fraction in the radiative study of our fluidized bed. Fig. 4 illustrate the time-dependence of the solids volume fraction from the CFD model (case H19N3.4).

\subsection{Stationary and transient calculation of radiative heat transfer}

The modeled repartition of the incoming light in each fluidized bed is compared to the experimental repartition of the three test conditions (Figs. 5-7). The radiative

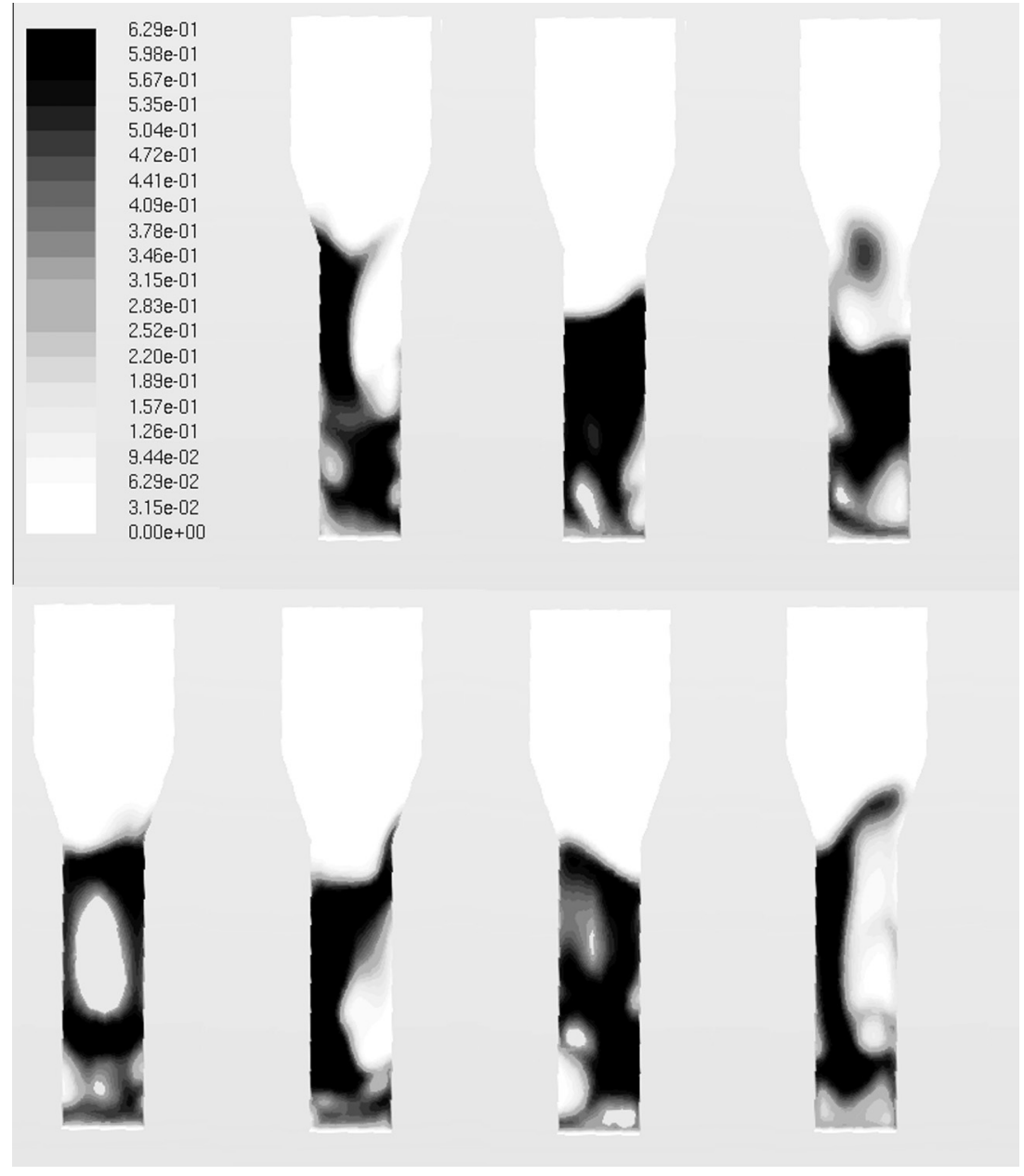

Fig. 4. Solid volume fraction fields from the CFD model taken at random times to illustrated the time-dependence of the solid volume fraction. 


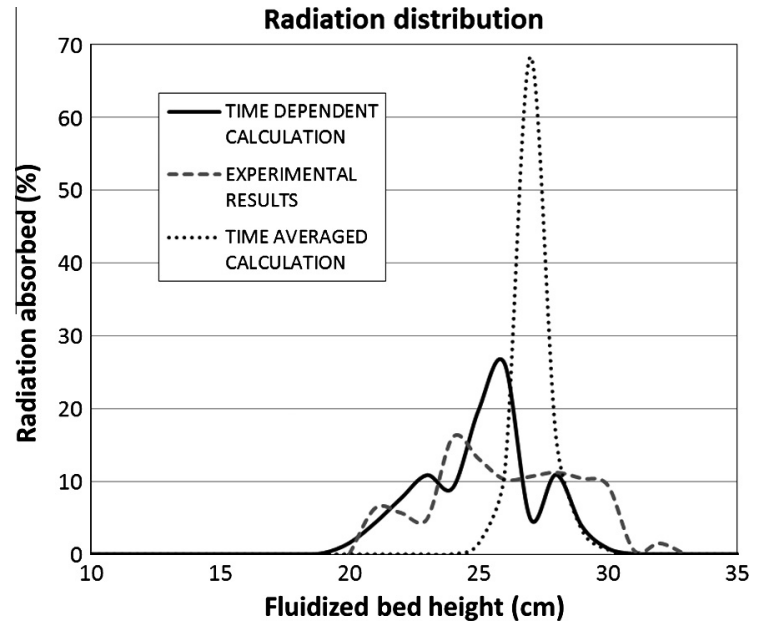

Fig. 5. Numerical versus experimental absorbed radiative flux in $1 \mathrm{~cm}$ wide slices of the case H19N3.4 fluidized bed receiver.

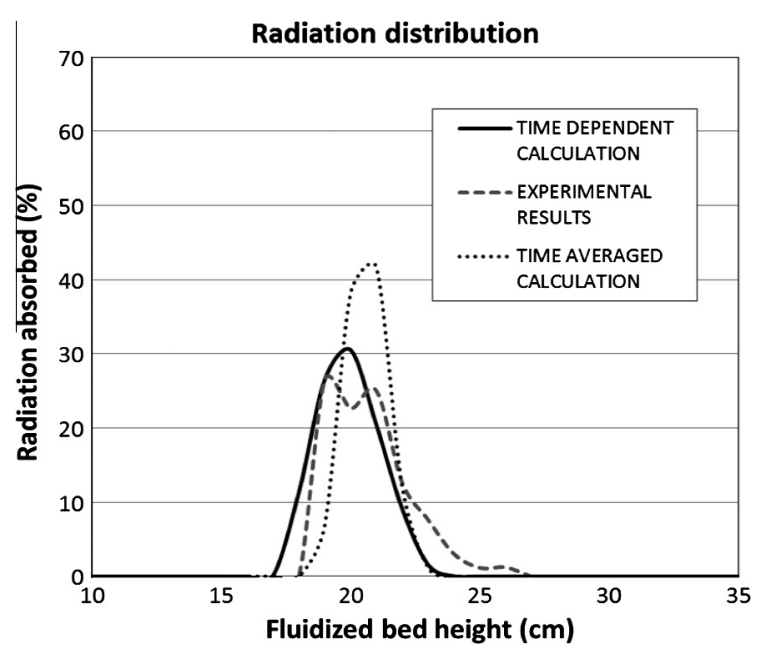

Fig. 6. Numerical versus experimental absorbed radiative flux in $1 \mathrm{~cm}$ wide slices of the case H16N2 fluidized bed receiver.

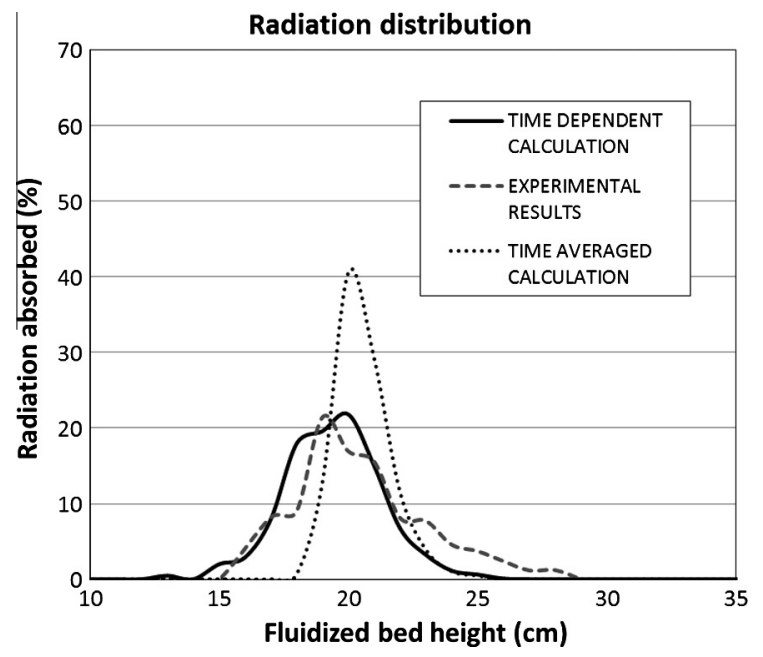

Fig. 7. Numerical versus experimental absorbed radiative flux in $1 \mathrm{~cm}$ wide slices of the case H12N3 fluidized bed receiver.

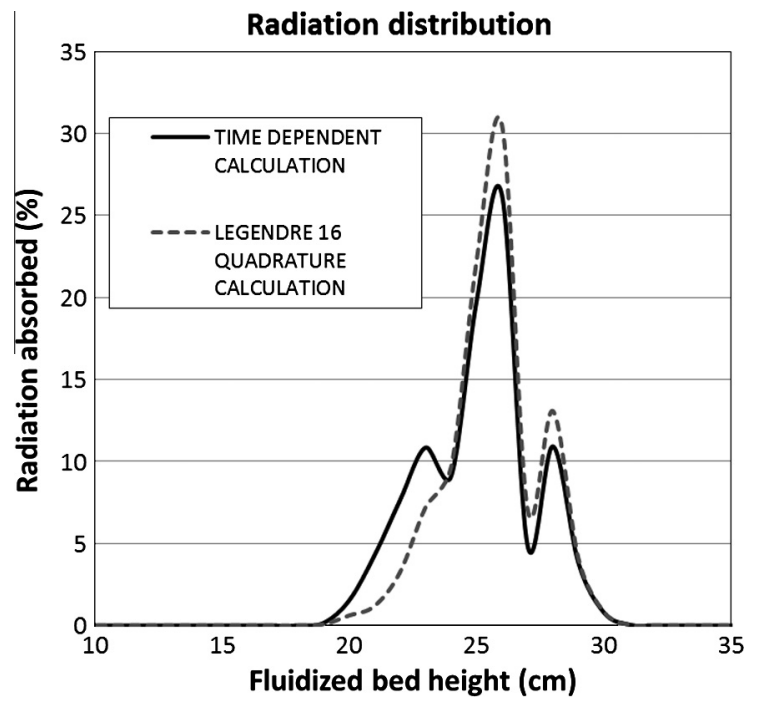

Fig. 8. Time-dependent and $k$-distribution calculations of the distribution of laser radiation for the case H19N3.4 bubbling fluidized bed.

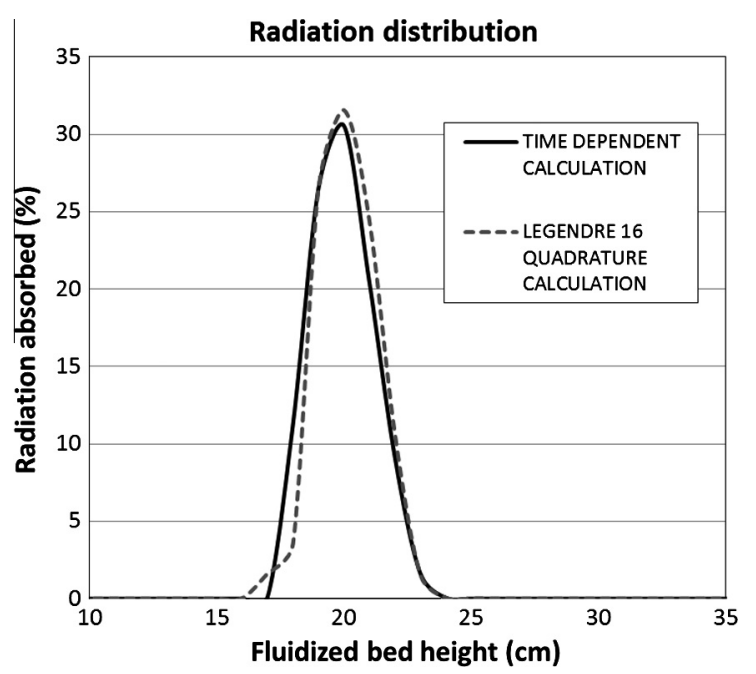

Fig. 9. Time-dependent and $k$-distribution calculations of the distribution of laser radiation for the case $\mathrm{H} 16 \mathrm{~N} 2$ bubbling fluidized bed.

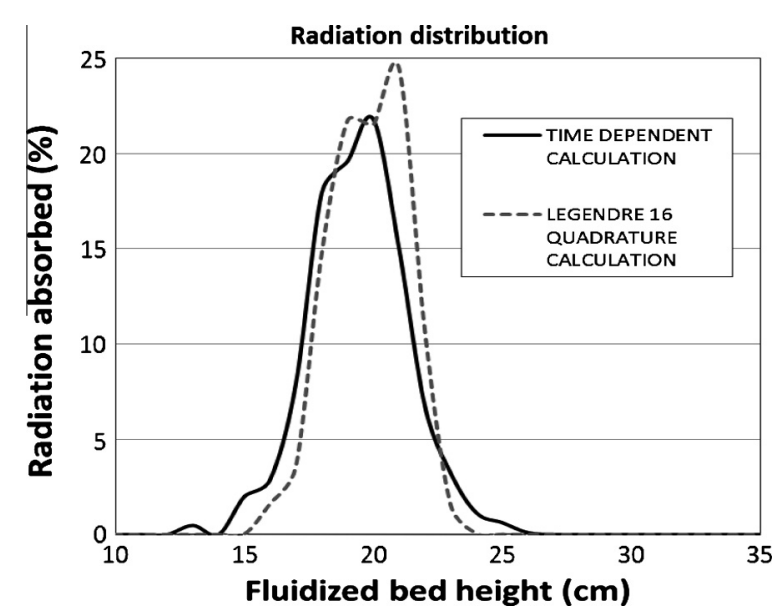

Fig. 10. Time-dependent and $k$-distribution calculations of the distribution of laser radiation for the case H12N3 bubbling fluidized bed. 


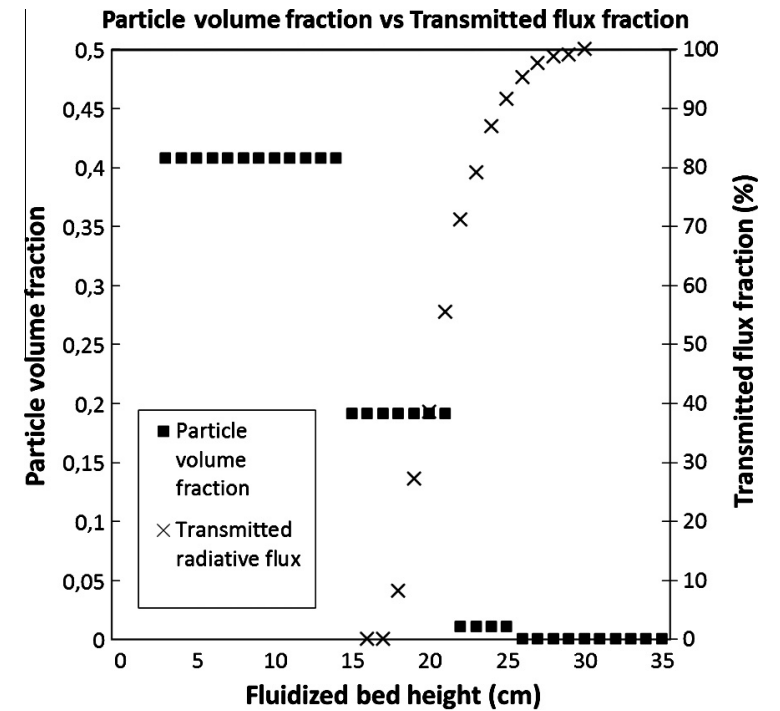

Fig. 11. Transmission of laser radiation and particle volume fraction for the case H19N3.4 fluidized bed receiver.

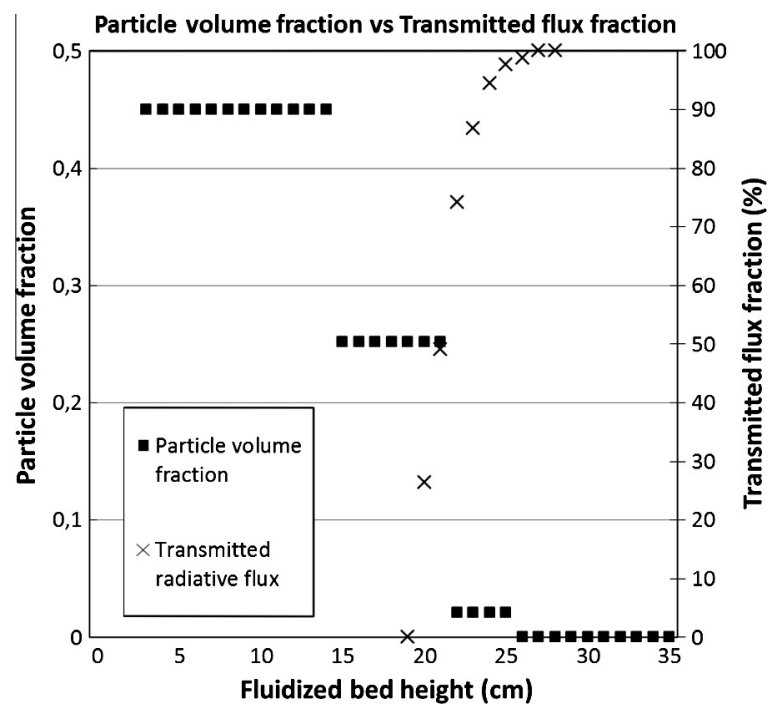

Fig. 12. Transmission of laser radiation and particle volume fraction for the case H16N2 fluidized bed receiver.

properties of the fluidized bed are calculated using timeaveraged or time-dependent CFD particle fields.

The experimental repartition of the radiation in the fluidized bed is spread over an area of more or less $10 \mathrm{~cm}$, localized between heights of $15-35 \mathrm{~cm}$. The maximal absorption per layer $(1 \mathrm{~cm}$ width) never exceeds $30 \%$ of the total incident radiation.

Numerical calculations based on the time-averaged particle volume fraction do not fit the experimental results. These calculations show overestimated values of the absorbed radiation in a smaller area of the fluidized bed. Thus, the averaged values are not representative of the absorbed radiation in the fluidized bed. The most significant difference is shown for the case H19N3.4 where the time-averaged calculation predicts a peak absorption of

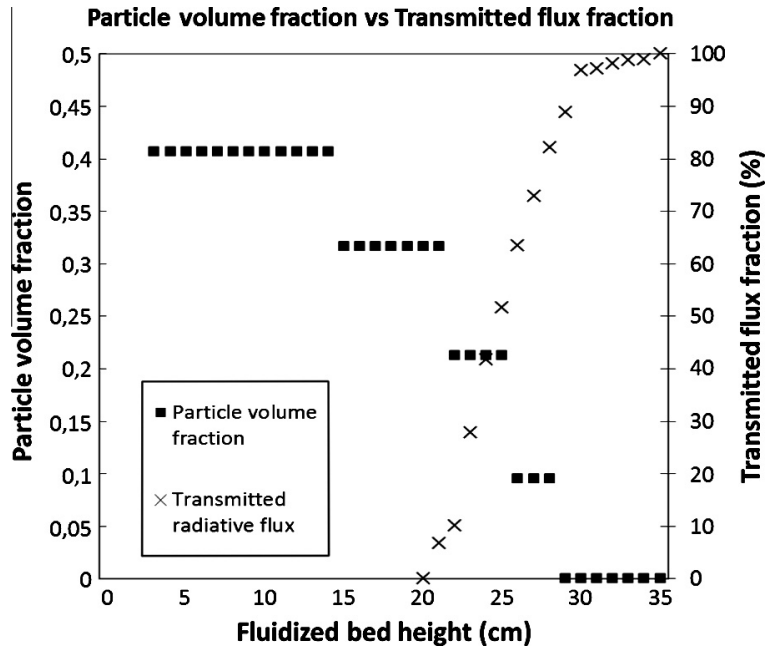

Fig. 13. Transmission of the laser radiation and particle volume fraction for the case H12N3 fluidized bed receiver.

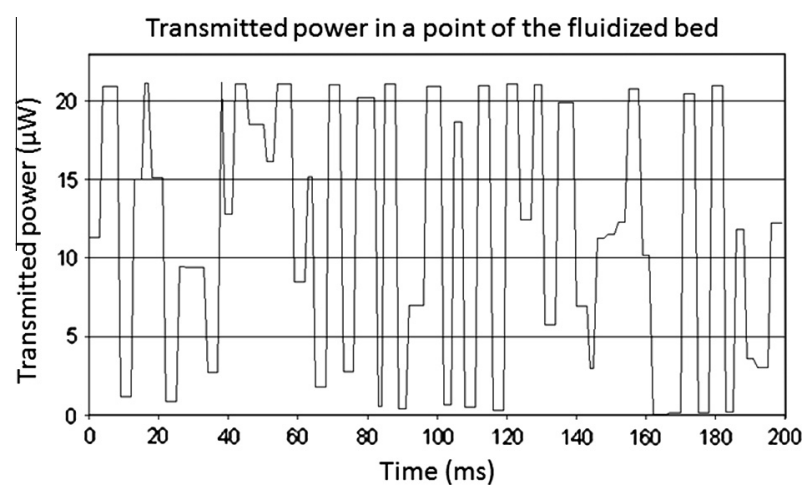

Fig. 14. Time-dependent evolution of the radiative power received at a random point in the fluidized bed, case H19N3.4.

$70 \%$ of the total incoming radiation in only one layer while the measured values never exceed $20 \%$ per layer. Although the penetration depth is reduced in the time-averaged calculations, the reduced depth is still $3 \mathrm{~cm}$ for the case H19N3.4.

Numerical calculations based on the time-dependent calculation of the particle volume fraction are in better agreement with the experimental results. Compared to the experimental results, the absorption is distributed in a similar manner across the fluidized bed. Nevertheless, we noticed an underestimation of the absorption coefficient within the first centimeters of the fluidized bed leading to a deeper absorption of radiation in the fluidized bed. This effect is probably due to small space-dependent phenomena (gas bubbles and particle clusters in the splash zone of the fluidized bed) which are not accurately described by our hydrodynamic model.

It is noticeable that predictions for case $\mathrm{H} 19 \mathrm{~N} 3.4$ are not as good as the predictions of the other two cases. We suppose that this is due to the position of the surface of fluidized bed H19N3.4 in the area where the column section increases. To overcome this problem, it is probably 

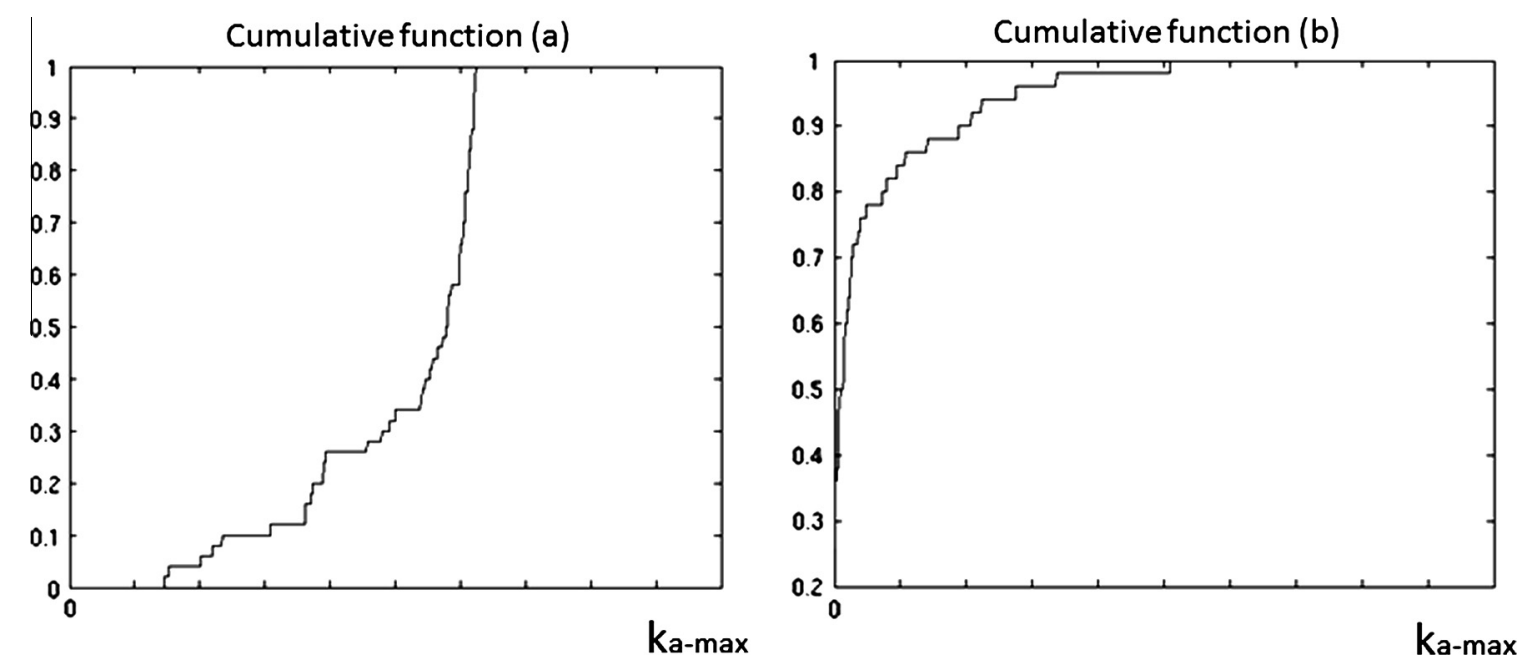

Fig. 15. Cumulative functions of the particle volume fraction for the case H19N3.4 measured (a) near the bottom and (b) near the top of the fluidized bed.

necessary to improve the CFD calculations to have a better simulation of the particles flow in the corners and near the walls of this complex area.

\subsection{Signal treatment using the $k$-distribution method}

We applied the $k$-distribution to the three test cases, comparing previous time-dependent calculations with calculations based on the spectral model (cf. Figs. 8-10). The time-dependent calculations are made of 100 instantaneous radiation distributions while the $k$-distribution calculations are made of the weighted average of only 16 radiation distributions calculated according to the Legendre quadrature points. Quadrature coefficients are calculated using the code KSPECTRUM developed by Eymet (2008) in the context of atmospheric studies.

The results demonstrate a good agreement between the time-dependent calculation and the $k$-distribution method, showing a calculation time divided by a factor 6 . Lower quadrature orders have been tested and while they prompted a more important reduction of computer time, they did so at the cost of a small decrease in precision.

\section{Discussion}

The results presented in this study demonstrate the importance of taking into account the time-dependent evolution of radiative properties within the bubbling fluidized bed to compute radiative transfer. This statement is corroborated by the experimental measurement of the extinction of the laser radiation in the fluidized bed (cf. Figs. 1113).

According to Figs. 11-13, the transmitted laser radiation can reach and cross zones where the optical extinction length, calculated from the time-averaged particle volume fraction, is less than one millimeter. This result shows that radiative properties that are calculated from time-averaged values of the particle volume fraction predict a shorter penetration depth of the radiation, which differs from experimental results. This is probably due to the strong non-homogeneity and turbulence of the medium. In the case of a bubbling fluidized bed, the transmission of radiation is strongly associated with the movement of the bubbles while the absorption of radiation is strongly associated with particle clusters ejected into the freeboard when a bubble bursts. The penetration of radiation far into the fluidized bed is a time-dependent phenomenon, as illustrated in Fig. 14. It shows raw experimental results of the radiative power received by the optical probe at a given point in the fluidized bed.

While we were relatively successful in predicting the time-dependent radiative properties of the fluidized bed using a CFD model, predicting the particle fraction in the freeboard of the fluidized bed is more difficult. The particle clusters that are propelled forward by the bursting bubbles are phenomena which are difficult to predict accurately by using an Eulerian model. As a consequence, the absorption of radiation by these clusters is miscalculated.

The numerical transmitted radiation, obtained using radiative properties averaged over the whole spectrum, fit well with the experimental results. While some wavelength dependent phenomenon does exist (mainly for wavelengths between 0.24 and $0.8 \mu \mathrm{m}$ ), our grey calculations are encouraging enough to think that our work can be extended in the field of broad-band concentrated solar energy applications. This model could later be improved by taking into account the spectral distribution of incident radiation and the wavelength dependent properties of $\mathrm{SiC}$ particles with more accuracy.

In this study, we proposed to treat the time-dependency of the absorption coefficient statistically using the same method as in the spectral properties of gases. The results are successful, showing reduced computer time and good modeling accuracy. However, we noticed small inaccuracies using the $k$-distribution method. This is probably due to the choice of the quadrature points which is difficult 
since the shape of the cumulative functions vary dramatically with regard to the position in the fluidized bed of the point that is being studied and also because the slope of these functions increases suddenly and dramatically (cf. Fig. 15). Performing a sensitivity analysis on quadrature types and orders could help us reduce the computer time and increase the precision of the calculation by choosing the points to better fit the cumulative functions at various locations in the fluidized bed.

\section{Conclusion}

In this study, we proposed a particular methodology to model the radiative heat transfer in a solar bubbling fluidized bed receiver by taking into account the time-dependent absorption and scattering of light in the particulate medium. This model uses the MCM as well as a timedependent field of optical properties that was predicted using a CFD tool implemented with an Eulerian model. An experimental set-up was also designed to validate the numerical predictions of the particle volume fraction and the penetration of radiation into the fluidized bed.

We showed that the experimental flux distribution inside the fluidized bed correlates well with the flux distribution computed from the time-dependent distribution of radiative properties while calculations using time-averaged radiative properties underestimate the penetration of the radiation into the fluidized bed. The CFD tool has proven reliable in predicting the radiative properties particularly of the lower and middle part of the fluidized bed. However, the prediction of the particle fraction in the freeboard of the fluidized bed is more difficult because the particle clusters propelled forward by the bursting bubbles are difficult to predict by using an Eulerian model. As a consequence, the absorption of radiation by these clusters in the freeboard is calculated with less accuracy. Finally, we proposed to apply a statistical treatment using the $k$ distribution method to the time-dependency of the radiative properties of a solar fluidized bed receiver. This allowed us to reduce computational time while providing good accuracy depending on the parameters of the quadrature used.

Even if high temperature solar experiments (Bounaceur, 2008) and numerical calculations (Baud, 2011) have shown that the penetration of the solar energy deep into the fluidized bed has a complex and limited effect on the performance of the receiver due to the high mixing rate of the particles in the fluidized bed, it have been shown that direct absorption of the solar energy on the walls create hot spots that limit the usability of this kind of receiver. For this reason, an interesting and straightforward application for this study could be to calculate the solar radiative fluxes on the walls of a receiver in order to predict the appearance of hot spots that can damage the receiver. In a future work, the $k$ distribution model, as it dramatically decreases computer time, could also be invaluable to study the sensitivity to the frequency of radiative calculations.

\section{References}

Ambartzumian, V., 1936. The effect of the absorption lines on the radiative equilibrium of the outer layers of the stars. Publ. Obs. Astron. Univ. Leningrad 6, 7-18.

Ansys. Fluent 12, 2009

Arancibia-Bulnes, C.A., Cuevas, S.A., 2004. Modeling of the radiation field in a parabolic trough solar photocatalytic reactor. Solar Energy 76 (5), 615-622.

Baud, G., 2011. Conception de récepteurs solaires à lit fluidisé sous flux radiative concentré. PhD thesis. INP de Toulouse.

Baud, G. et al., 2012. Radiative heat transfer modelling in a concentrated solar energy bubbling fluidized bed receiver using the Monte Carlo Method. J. Phys.: Conf. Ser. 369, 012030.

Bounaceur, A., 2008. Intéraction lit fluidisé de particules solides-rayonnement solaire concentré pour la mise au point d'un procédé de chauffage de gaz à plus de 1000 K, Ph.D. thesis. Ecole Nationale Supérieure des mines de Paris.

Chaouki, J., Cui, H., Sauriol, P., 2003. High temperature fluidized bed reactor: measurement, hydrodynamics and simulation. Chem. Eng. Sci. 58, 1071-1077.

Chen, G., Andries, J., Spliethoff, H., Fang, M., van de Enden, P.J., 2004. Biomass gasification integrated with pyrolysis in a circulating fluidised bed. Solar Energy 76 (1-3), 345-349.

Coelho, P., Perez, P., El Hafi, M., 2003. Benshmark numerical simulation for radiative heat transfer in two-dimensional axisymetric enclosures. Num. Heat. Trans. Part B 43, 425-444.

De Lataillade, A., Dufresne, J.L., El Hafi, M., Eymet, V., Fournier, R., 2002. A net exchange Monte Carlo approach to radiation in optically thick systems. J. Quant. Spectrosc. Radiat. Transf. 74, 563-584.

Delatorre, J., Baud, G., Bézian, J.J., Blanco, S., Caliot, C., Cornet, J.F., Coustet, C., Dauchet, J., El Hafi, M., Eymet, V., Fournier, R., Gautrais, J., Gourmel, O., Joseph, D., Meilhac, N., Pajot, A., Paulin, M., Perez, P., Piaud, B., Roger, M., Rolland, J., Veynandt, F., Weitz, S., 2013. Monte Carlo advances and concentrated solar applications. Solar Energy (18 May).

Di Felice, R., 1994. The voidage functions for fluid particule interaction systems. Int. J. Multiphase Flow 20, 153-159.

Donnadieu, G., 1992. Transmission de la chaleur dans les milieux granulaires. Revue de l'Institut Français du Pétrole XVI (9), 952-976.

Eymet, V., 2008. KSPECTRUM < http://code.google.com/p/kspectrum/>.

Flamant, G., Olalde, G., 1983. High temperature solar gas heating comparison between packed and fluidized bed receivers-I. Solar Energy 31 (5), 463-471.

Furosaki, S., Kai, T., 1985. Behaviour of fluidized beds of small particles at elevated temperatures. J. Chem. Eng. Japan 18, 113-118.

Gordillo, E.D., Belghit, A., 2010. A two phase model of high temperature steam-only gasification of biomass char in bubbling fluidized bed reactors using nuclear heat. Int. J. Hydrogen Energy 36, 374-381.

Haddad, I.M., Elsayed, M.M., 1988. Transient performance of fluidized bed solar receiver at various parametric conditions. Solar Wind Technol. J. 5 (6), 653-659.

Handbook of optical constants of solids. Academic Press, 1985.

Hua, Y., Flamant, G., Lu, J., Gauthier, D., 2004. 3D modelling of radiative heat transfer in circulating fluidized bed combustors: influence of the particulate composition. Int. J. Heat Mass Transfer 48, 1145-1154.

Joseph, D., Perez, P., El Hafi, M., Cuenot, B., 2009. Discrete ordinates and Monte Carlo methods for radiative transfer simulation applied to CFD combustion modeling. J. Heat Transfer 131-5, 052701.

Klein, Hanna Helena, Karni, Jacob, Ben-Zvi, Rami, Bertocchi, Rudi, 2007. Heat transfer in a directly irradiated solar receiver/reactor for solid-gas reactions. Solar Energy 81 (10), 1227-1239.

Kunii, D., Mii, T., Yoshida, K., 1973. Temperature effect on the characteristics of fluidized beds. J. Chem. Eng. Japan 6, 100-102.

Lathouwers, D., Bellan, J., 2001. Modeling of dense gas-solid reactive mixtures applied to biomass pyrolysis in a fluidized bed. Int. J. Multiphase Flow 27 (12), 2155-2187. 
Lun, C.K.K., Savage, S.B., Jeffrey, D.J., Chepurniy, N., 1984. Kinetic theories for granular flow: inelastic particles in Couette flow and slightly inelastic particles in a general flow field. J. Fluid Mech. 140, 223-256.

Mancuso, L., Formisani, B., Girimonte, R., 1997. Analysis of the fluidisation process of particle beds at high temperature. Chem. Eng. Sci. 53 (5), 951-961.

Mendes, A., Dollet, A., Ablitzer, C., Perrais, C., Flamant, G., 2008. Numerical simulation of reactive transfers in spouted beds at high temperature: application to coal gasification. J. Anal. Appl. Pyrolysis $82,117-128$.

Metropolis, N., Ulam, S., 1949. The Monte Carlo Method. J. Am. Statistical Assoc. 44 (247), 335-341.

Mie, G., 1908. Beitrage zur optik truber medien spiezell kolloidallen metallosungen. Ann. D. Phys. 25, 377-445.

Muller, R., Zedtwitz, P.V., Wokaun, A., Steinfeld, A., 2003. Kinetic investigation on steam gasification of charcoal under direct high-flux irradiation. Chem. Eng. Sci. 58, 5111-5119.

Rensner, D., Werther, J., Hartge, E.U., 1986. Measuring techniques for gas/solid fluidized bed reactors. Chem. Eng. Tech. 58, 688-689.

Reuge, N., Cadoret, L., Caussat, B., 2009. Multifluid Eulerian modelling of a silicon fluidized bed chemical vapor deposition process: analysis of various kinetic models. Chem. Eng. J. 148, 506-516.
Schaeffer, D.G., 1987. Instability in the evolution equations describing incompressible granular flow. J. Diff. Eq. 66, 19-50.

Selçuk, N., Batu, A., Ayranci, I., 2002. Performance of method of lines solution of discrete ordinates method in the freeboard of a bubbling fluidized bed combustor. J. Quant. Spectrosc. Radiat. Transf. 73, 503-516.

Sinclair, J.L., Jackson, R., 1989. Gas-particle flow in a vertical pipe with particle-particle interactions. AIChE J. 35, 1473-1486.

Syamlal, M., Rogers, W., O'Brien, T.J., 1993. MFIX Documentation: Volume 1, Theory Guide. National Technical Information Service. Springfield, VA.

Tien, C.L., Drolen, B.L., 1987. Thermal radiation in particulate media with dependent and independent scattering. Annu. Rev. Num. Fluid Mech. Heat Transfer 1, 1-32, Hemisphere, New York.

Trommer, D. et al., 2005. Hydrogen production by steam-gasification of petroleum coke using concentrated flux solar power: thermodynamic and kinetic analyses. Int. J. Hydrogen Energy 30, 605-618.

Von Zedtwitza, P., Lipinskia, W., Steinfeld, A., 2006. Numerical and experimental study of gas-particle radiative heat exchange in a fluidized-bed reactor for steam-gasification of coal. Chem. Eng. Sci. 62, 599-607. 